\title{
Influence of Mechanical and Thermal Material Properties on Laser-Produced Crater-Morphology and their Study by Focused Ion Beam \& Scanning Electron Microscope Imaging
}

\author{
D. Bigoni ${ }^{*}$, M. Milani ${ }^{*}$, R. Jafer ${ }^{* *}$, C. Liberatore ${ }^{* *}$, S. Tarazi ${ }^{* *}$, L. Antonelli ${ }^{* *}$ and D. Batani ${ }^{* *}$ \\ *Department of Material Science and FIB-SEM 'Bombay'Lab, \\ University of Milano-Bicocca, Via Cozzi 53, 20125 Milan, Italy \\ ** Department of Physics “G.Occhialini”, University of Milano-Bicocca, \\ Piazza della Scienza 3, 20126 Milan, Italy \\ Email:batani@mib.infn.it
}

\begin{abstract}
Ablation of material can be obtained by high intensity laser beams and resulting in crater formation in samples. Crater morphology is due to concurring processes: subsurface vaporization, heterogeneous or/and homogeneous boiling, subsurface heating, spherical shock wave propagation, and the volume of craters is expected to be inversely proportional to the bulk modulus of target material. FIB-SEM dual beam system can be used to investigate the role of mechanical and thermal material properties in the laser produced crater morphology. In particular, FIB-SEM images of craters in conductive samples show a higher deposition around craters than in insulators, due to the different thermal conductivity of materials. Due to subsurface modifications on samples, both circular and noncircular craters may be formed. Therefore the analysis of the profile of these structures can be used to back up at dissimilar laser ablation processes

DOI: $10.2961 / \mathrm{jlmn} .2010 .02 .0013$
\end{abstract}

Keywords: pulsed laser, ablation, crater formation, bulk modulus, thermal coefficients, focused ion beam imaging, scanning electron microscope

\section{Introduction}

Focusing a high intensity laser beam on a solid target activates many effects: ablation of material (hole drilling, surface modifications); emission of radiation (which can be used for plasma and material diagnostics); re-deposition of the ablated material on a surface (PLD: pulsed laser deposition). These effects are used in several fields, such as micromachining, surgery, $\mathrm{x}$-ray laser generation, mass spectrometry of bio-molecules, art cleaning/restoration, and fundamental physics studies. Advantages of using laserbased techniques include economic ones (reduction of work time; increase in production quality) but above all technological advantages: laser machining is precise (the beam can be focused on extremely small areas), clean and silent. Also, the laser is a non contact instrument that guarantees: absence of mechanical pressure on the piece; working capability independent of hardness of the material; capability of cutting coated or surface-treated material; no contact contamination of materials, no wear on the laser itself. Laser cutting also allows a high degree of automation and flexibility offering easy integration with other automated systems and capability of adapting to changes in production requirements [1].

Laser ablation is a multi step process. In the first step, the laser beam energy is transferred to the (free) electrons of the conduction band. More electrons gain energy and are extracted from the material thus overcoming the extraction work of material. Third, the local lattice temperature increases, leading to the chemical bonds breakdown. Fourth, ablation of the material and formation of a plasma plume takes place, generating a crater on the material surface $[1,2,3]$.

In addition, for insulating materials, a preliminary phase takes place during which free electrons are created. In particular, ionization occurs because of two simultaneous mechanisms: the absorption of multiple photons and the ionization by the electron impact (avalanche ionization). The relative contribution of both mechanisms depends on laser wavelength, pulse duration, intensity and the atomic number of the sample [2].

Unlike ion motion, energy transfer by heat conduction starts immediately after energy absorption. Therefore a heat wave can propagate outside the heated area, followed at later times by a shockwave propagating in the cold material and gradually dissipating its energy that transforms into a sound wave due to the work done against the internal pressure of the material (Young's Modulus, Y). The distance at which the shock wave changes into a sound wave defines the shock affected volume, as shown in [1,2], and the whole process produces a "crater" in strong analogy with those generated, for instance, by meteorite impact on the moon or the hearth. In particular, laser crater morphology depends on cooling through simultaneous thermal processes: subsurface vaporization; heterogeneous boiling (migration of particles from the condensed phase into the volatile phase through the formation of bubbles for the presence of heterogeneous nuclei in the irradiated zone); homogeneous boiling (explosive boiling of target material occurring 
close to the critical temperature, with a consequent release of a mixture of vapour and droplets sometimes termed as "gas of droplets ejection" or "phase explosion"); subsurface heating. Blainer et al. [4] have classified them as a function of the laser pulse duration (as shown in tab. 1). Therefore with $\tau$ in the ps range, the prevalent active process is the vaporization of material.

The study of crater morphology can bring information on thermal processes taking place in the material, on shockwave dynamics, etc., and consequently on structures and morphology. The use of novel imaging machines and techniques at the sub-um scale is therefore important. One of such machines is the FIB-SEM microscopy system, already recently used for studying the laser ablation [5-7].

In this paper, FIB-SEM imaging will be used to investigate the role of mechanical and thermal properties of materials in determining laser crater size and morphology.

Table 1 Processes in laser ablation vs. pulse duration $(\tau)$

\begin{tabular}{|l|l|}
\hline LASER ABLATION PROCESSES & $\tau$ \\
\hline Subsurface vaporization & $f s-n s$ \\
\hline Heterogeneous boiling & $<50 n s$ \\
\hline Homogeneous boiling & $<1 \mu s$ \\
\hline Subsurface heating & $\sim \mu s$ \\
\hline
\end{tabular}

\section{Materials and Methods}

A Nd:YAG (pulse duration $50 \mathrm{ps,} \mathrm{wavelength} 532 \mathrm{~nm}$, laser power $600 \mathrm{MW}$, energy density up to $\approx 1500 \mathrm{~J} / \mathrm{cm}^{2}$ ) has been used to irradiate samples and also to drill through materials: $\mathrm{Al}, \mathrm{Si}, \mathrm{SiO}_{2}$, transparent polypropylene (PP), white polyethylene (WPE) and black polyethylene (BPE). Irradiation of aluminum has been made both in air and in vacuum, while other samples were irradiated in vacuum only. Vacuum operation allows to exclude laser-air coupling that could reduce ablation efficiency. The laser ablation set-up is shown in fig. 1 .

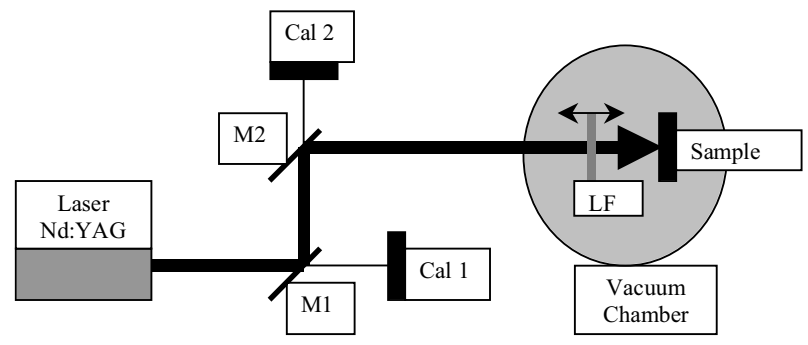

Fig. 1 Scheme of the experimental set-up

The laser beam hits the first mirror (M1), that reflects it on the second mirror (M2), whose role is to direct the beam in the centre of the focal lens (LF) inside of the interaction chamber. The lens has a focal length of $10.4 \mathrm{~cm}$ and is used to focus the laser beam on sample. It is assembled on a motorized mount, which allows to change the lens-sample distance under vacuum. Two calorimeters (see fig.1) monitored the laser beam energy on each laser shot. In the experiment, we drilled through various samples by irradiating them with a high number of shots on the same position (with the laser working at $2 \mathrm{~Hz}$ pulse frequency), and stopped irradiation exactly at this time as soon as the hole was obtained. A He-Ne laser beam, collinear to the main beam through the focusing lens, served for alignment and also for indicating when the hole was obtained (by shining through it). It's clear that while for non-transparent materials one sees a drastic change from no signal to a non-zero signal, instead in the case of transparent materials one rather sees an increase of the transmitted signal [let's notice however that after the $1^{\text {st }}$ shots the amount of transmitted light from the He-Ne is drastically reduced due to the processes of crater formation themselves, including surface destruction and induced opacity of the sample].

FIB-SEM microscope incorporates a focused ion beam and an electron beam in high vacuum for imaging and micromachining of bulk or structured (soft) materials. The scanning electron microscope (SEM) is based on a tungsten electron column, able to operate both as a conventional, high vacuum SEM and as a low vacuum or "Environmental" SEM, which allows working pressures up to 3000 $\mathrm{Pa}$ thanks to special gas detectors. The Focused Ion Beam (FIB) is a tool that performs basically three functions: ion imaging (from secondary electrons or ions), milling (precision down to $10 \mathrm{~nm}$ ) and material deposition [7, and refs therein].

The crater morphology of silicon and aluminum samples has been investigated using the FIB/SEM dual beam (FEI, Quanta 3D) in high vacuum condition at different electron acceleration and current, for sake of comparison of different samples. The best parameters were selected to be with $20 \mathrm{keV}$ primary electrons and $81 \mathrm{pA}$ current. Glass and other silicon laser craters have been analyzed by scanning ion microscope in high vacuum mode with $30 \mathrm{keV}$ primary ions and 30pA current. Polymer craters have been observed by SEM images in low vacuum mode, to bypass the charging effect of the insulator samples, with $5 \mathrm{keV}$ and $90 \mathrm{pA}$. All these images have been collected using secondary electrons.

\section{Results}

Notice that, in principle, it is known that for given laser parameters the averaged ablation depth per laser shot is sample thickness dependent. For thicker samples or deeper holes, one can expect that it should be more difficult for the ablated material/plume to escape from the ablation site or there would be more ablated material falling back to the holes, and also the effective laser fluence would become smaller as the ablation moves deeper. To have a fair comparison of laser ablation of different materials, it would therefore be better to have samples of the same thickness but unfortunately, in our experiment, we could not find samples of the different materials with the same thickness. However when we compare the ablated depth per shot with the sample thickness, we find no correlation. This shows that although present, the effect described above is probably not predominant in our conditions. Similarly, by irradiating (plastics) samples of the same material but different thickness, we did not find any significant variation of the ablated depth per shot under our experimental conditions [notice however that we used a few samples only and only with thickness larger than the depth of focus of our laser system and focusing lens, i.e. $\approx 200 \mu \mathrm{m}$ ) 
Table 2 Number of laser shots needed to drill through each irradiated sample, crater volume and depth, removed depth per laser shot and removed crater volume per laser shot.

\begin{tabular}{l|l|l|l|l|l}
\hline $\begin{array}{l}\text { Sam } \\
\text { ples }\end{array}$ & $\begin{array}{l}\text { Sample } \\
\text { depth } \\
(\mu \mathrm{m})\end{array}$ & $\begin{array}{l}\mathrm{n}^{\circ} \\
\text { shots }\end{array}$ & $\begin{array}{l}\text { Crater Vol } \\
\left(\times 10^{6}\right. \\
\left.\mu \mathrm{m}^{3}\right)\end{array}$ & $\begin{array}{l}\text { Depth/ } \\
\text { shot } \\
(\mu \mathrm{m})\end{array}$ & $\begin{array}{l}\text { Volume per } \\
\text { shot }\left(\times 10^{6}\right. \\
\left.\mu \mathrm{m}^{3}\right)\end{array}$ \\
\hline $\mathrm{BPE}$ & $770 \pm 10$ & $144 \pm 2$ & $30 \pm 0.7$ & $5.3 \pm 0.1$ & $0.21 \pm 0.008$ \\
\hline $\mathrm{WP}$ & $770 \pm 10$ & $135 \pm 2$ & $42 \pm 0.9$ & $5.7 \pm 0.15$ & $0.31 \pm 0.01$ \\
$\mathrm{E}$ & & & & & \\
\hline $\mathrm{PP}$ & $420 \pm 10$ & $68 \pm 2$ & $8.8 \pm 0.3$ & $6.4 \pm 0.3$ & $0.13 \pm 0.008$ \\
\hline $\mathrm{Si}$ & $680 \pm 10$ & $67 \pm 2$ & $1.1 \pm 0.07$ & $10.1 \pm 0.4$ & $0.016 \pm 0.001$ \\
\hline $\mathrm{Al}$ & $100 \pm 10$ & $57 \pm 2$ & $0.065 \pm 0.01$ & $1.8 \pm 0.2$ & $0.0011 \pm 0.0002$ \\
\hline $\mathrm{SiO}_{2}$ & $150 \pm 10$ & $22 \pm 2$ & $0.8 \pm 0.07$ & $7 \pm 1$ & $0.04 \pm 0.007$ \\
\hline
\end{tabular}

Errors listed in Tab. 2 have been determined by applying the laws of error propagation and by considering:

the uncertainty of micrometer $( \pm 10 \mathrm{um})$ used to measure the sample thickness;

the fact that working with the laser at a repetition frequency of $2 \mathrm{~Hz}$, and assuming a reaction time of $0.5 \mathrm{~s}$ (clearly an overestimation) in stopping the laser system after the laser has drilled through the sample.
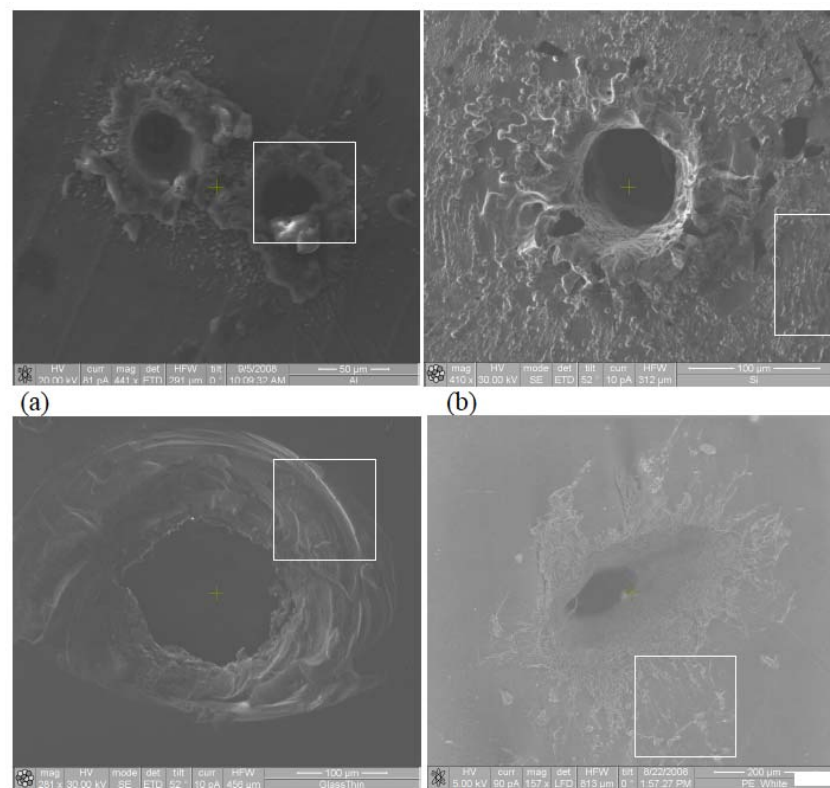

(b)
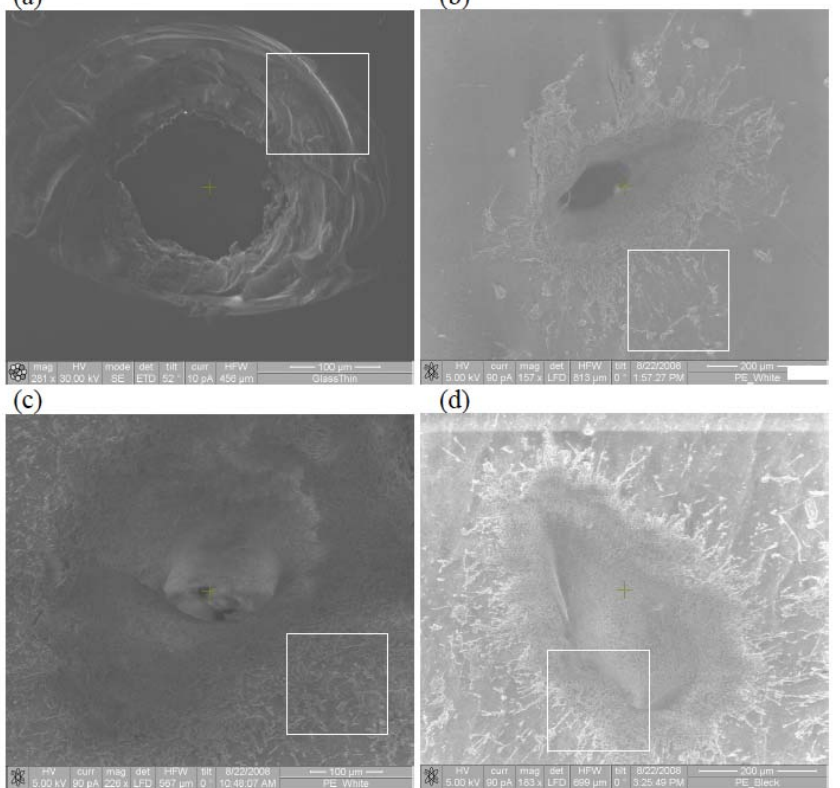

(e)

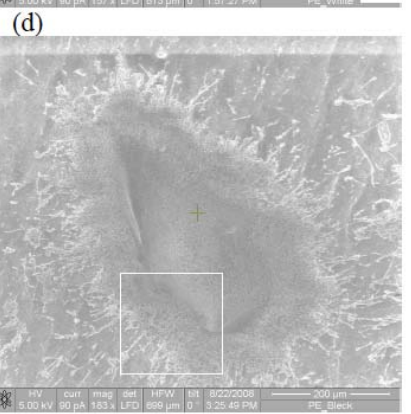

(f)

Fig. 2 FIB-SEM images of laser craters: (a) Al; (b) Si; (c) glass; (d) transparent polypropylene; (e) white polyethylene (WPE); (f) black polyethylene (BPE). The square underlines the morphology around to crater. These images show the entrance side of the through-holes in the samples.
Fig. 2 shows the FIB-SEM images of the laser craters in the different materials. In particular, craters in silicon and aluminum have a similar circular shape (Fig. 2a and b) [notice that the two craters in Fig. 2 are simply the results of two successive irradiation of the sample with the same laser parameters]. Instead, in insulator samples, the shape of craters is circular only for glass (Fig. 2c). For polymeric samples, instead, it shows a pear shape, despite the fact that the irradiation beam profile was the same. Also, the amount of deposited material around the hole is higher in the conductive samples (Al, Si) than in insulating ones. This shows that the crater morphology changes as a function of material properties.

The SEM image of the fig. 2d), e), and f) respectively show the crater generated in transparent polypropylene, white polyethylene, and black white polyethylene, all showing similar morphologies in that one we can observe the same morphology of the polyethylene crater. In particular, an elliptical hole surrounded by pear-shaped crater can be observed. Again, the deposited amount of material around the crater surface is very low: we can only observe some surface modifications, that seem to reproduce the "splashing effect" due to material processes like heterogeneous and homogeneous boiling induced by the laser around the focal spot (where the laser intensity is smaller). These effects are clearer in the silicon crater, whose thermal conductivity is larger than for the polymer samples (as reported in Tab. 3) and as a consequence the formation of the surface bubbles is favored.

Fig. 3 shows the same images of Fig. 2 with the orthogonal two axis scales of the crater clearly evidenced. In the case of silicon, the major axis is shorter than in the case of black polyethylene, polypropylene, and o white polyethylene, and the crater shape is more circular despite the fact that the thickness of the silicon samples is similar to the one of the plastics samples (in all cases we verified that here is no correlation between the aspect ratio of the crater and the thickness of the sample). Therefore, again, the thermal and mechanical properties of the material seem to play a role.

Let's also notice that the aluminum crater (Fig. 3a) has a circular shape and its diameter is $\approx 50 \mu \mathrm{m}$, quite close to the focal spot diameter. [Hence it could be possible to use the crater size in some materials (as Al) as a diagnostics of the laser focal spot width, of course when a low pulse energy, just above the ablation threshold, is used. This is not possible in the case of other materials, where craters are much larger than the spot.]

\section{Comments and discussion}

Crater volumes have been calculated from Fig. 3, by assuming a conical shape with elliptical base $(\mathrm{V}=\pi \mathrm{abh} / 3)$. The height $h$ of the crater was equal to the thickness of the sample (since we drilled through it).

Let's notice that according to [8], the evaluation of the ablated volume (coupled to regression techniques) is the most reliable way to evaluate the laser ablation parameters (e.g., for determining the ablation rate, ablation threshold, etc.). In this context, they proposed AFM (Atomic Force Microscope) as a tool to measure the crater volume. However, due to the working limitations of AFM in the verticaldirection (allowing a few microns depth at most), the use of 
FIB/SEM device is indeed an interesting alternative for the evaluation of volume of deeper craters.
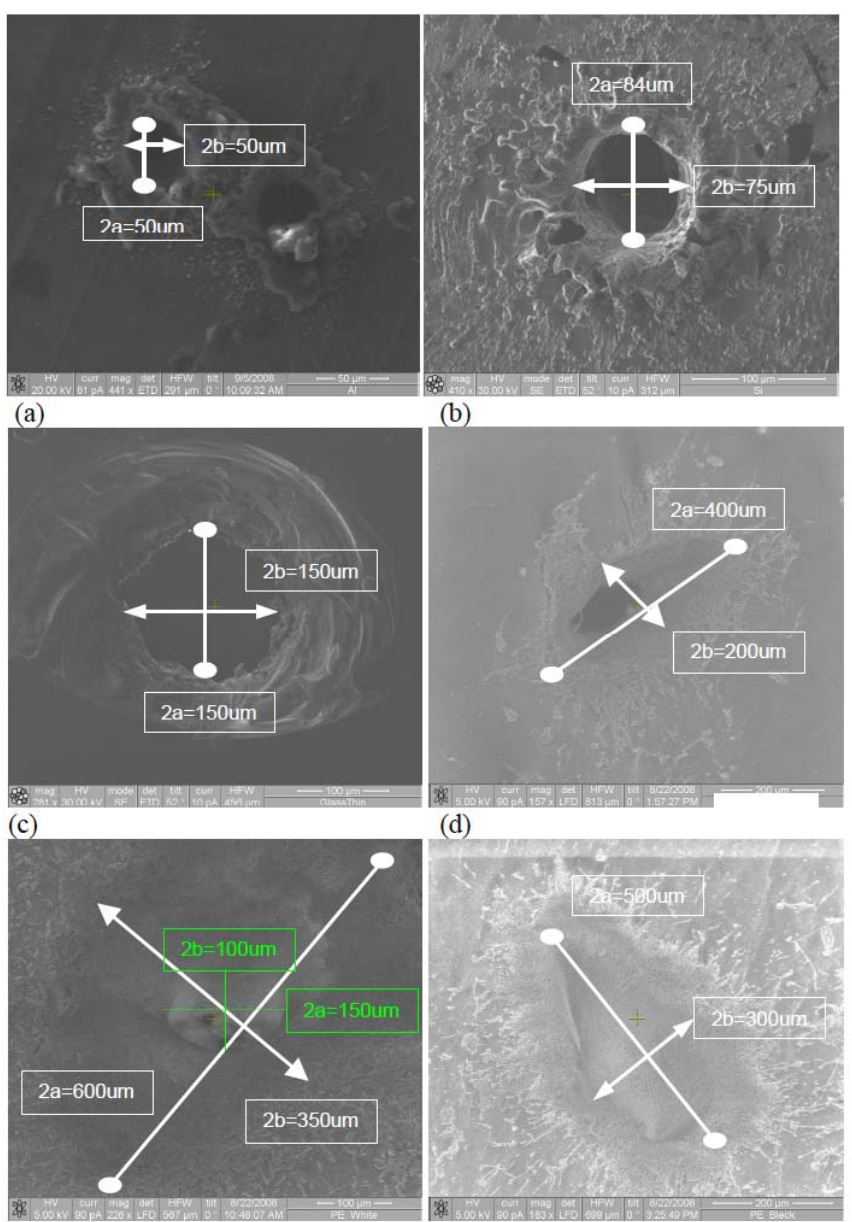

(b)
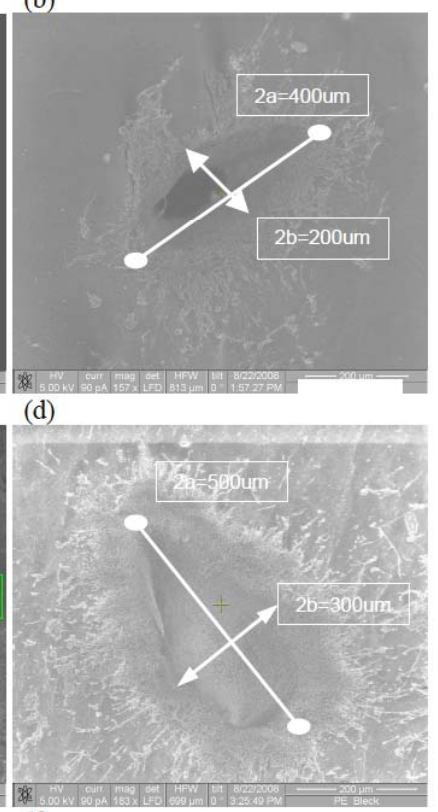

(e)

(f)

Fig. 3 FIB-SEM images of laser craters: (a) Al; (b) Si; (c) glass; (d) transparent polypropylene; (e) white polyethylene (WPE); (f) black polyethylene (BPE). Lines show how we have measured the crater area.

Tab 2 shows the number of shots needed for drilling through each sample, as well as the crater volume, and the crater depth and volume of matter ablated per single shot. We have then tried to correlate the characteristics of ablation and craters to thermal and mechanical properties of the samples. Tab. 3 shows the bulk modulus, thermal expansion and thermal conductivity for each material [9 - 11].

Table 3 Thermal $/$ mechanical properties of irradiated samples

\begin{tabular}{l|l|l|l}
\hline Sample & $\begin{array}{l}\text { Bulk } \\
\text { Modulus } \\
(\mathbf{G P a})\end{array}$ & $\begin{array}{l}\text { Linear Thermal } \\
\text { Expansion Coeffi- } \\
\text { cient }\left(\times \mathbf{1 0}^{5}{ }^{\circ} \boldsymbol{C}^{-1}\right)\end{array}$ & $\begin{array}{l}\text { Thermal Con- } \\
\text { ductivity } \\
(\boldsymbol{W} / \mathbf{m K})\end{array}$ \\
\hline $\boldsymbol{B P E}$ & 1.38 & 18 & $0.42-0.51$ \\
\hline $\boldsymbol{W P E}$ & 1.38 & 23 & $0.42-0.51$ \\
\hline $\boldsymbol{P P}$ & $1.5-2$ & 12 & $0.1-0.22$ \\
\hline $\mathbf{S i}$ & 130.91 & 3 & 163 \\
\hline $\boldsymbol{A l}$ & 76 & 24 & 237 \\
\hline $\mathbf{S i O}_{\mathbf{2}}$ & 75 & 9 & 1.005 \\
\hline
\end{tabular}

First of all, the theory developed by Gamaly, Tikhoncuk, et al. [2] directly relates the crater radius to the cubic root of the bulk modulus of the material as

$$
r_{\text {crater }}=\sqrt[3]{\frac{E_{a b s}}{\frac{4}{3} \pi Y}}
$$

which comes from the condition that for $r<r_{\text {crater }}$, the internal energy in the volume within the shock front, given by the absorbed laser energy, $\mathrm{E}_{\mathrm{abs}} \sim 4 / 3\left(\pi \mathrm{Yr}_{\text {crater }}{ }^{3}\right)$, is comparable to the bulk modulus, or Young's modulus, $\mathrm{Y}$ of the material. In other words a crater is created if the pressure in absorption volume significantly exceeds the Y of the solid.

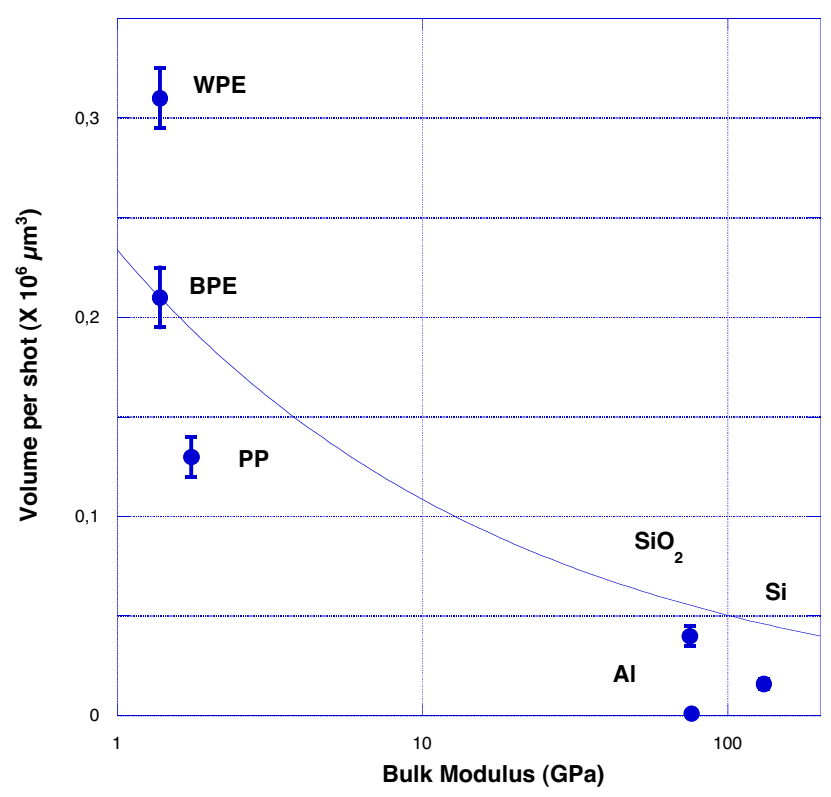

Fig. 4 Crater volume per laser shot vs. bulk modulus of the irradiated matter. The continuous line is the plot of the cubic root in eq. (1) and does not represent a fit to data.

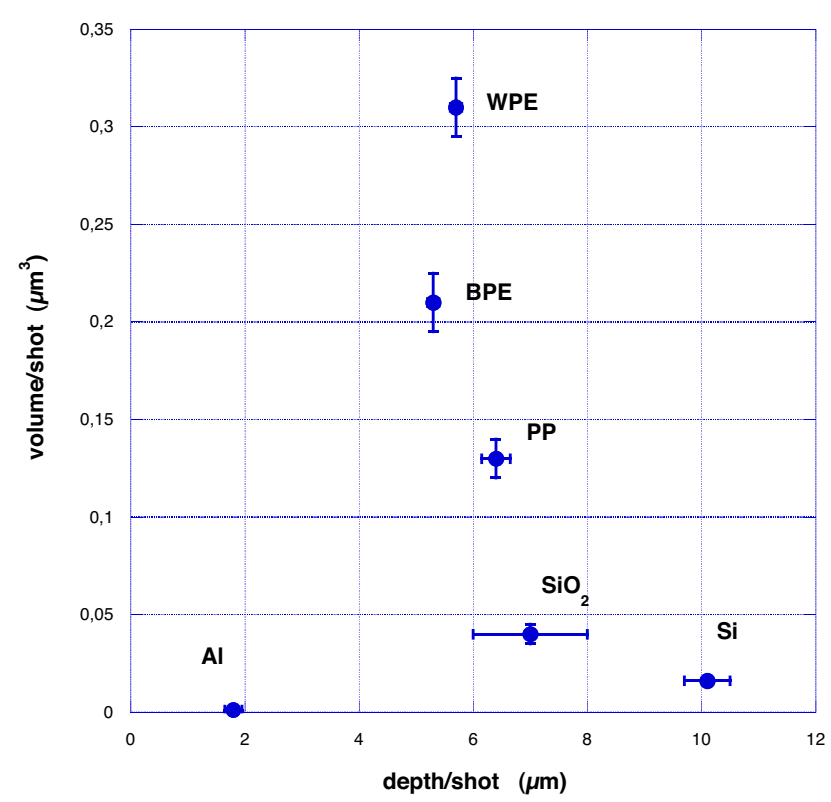

Fig. 5 Crater volume per laser shot as function of the removed depth per laser shot.

This is similar to Sedov's self-similar theory of pointlike blasts [14]. The result is shown in Fig. 4. Notice that 
the continuous line in Fig. 4 corresponds to the above formula (without any free parameters) and it is not an interpolation of experimental results!

We see that the general trend of experimental data is fairly well reproduced by the formula. However there is a large scattering of data. In addition, the qualitative analysis of the pictures obtained by FIB/SEM, and shown in Fig.2, suggests that there are other types of morphological changes (and not only the crater size) when one moves from one material to the other. Finally plotting the ablated volume per shot vs. the ablated depth per shot, we find a non-constant behavior (shown in Fig. 5). All this suggests that indeed some other properties of the irradiated material, apart from its bulk modulus, is also playing a role in determining the crater size and crater morphology.

In Fig. 5, we also see that the general trend, among the samples we have analyzed, points to a reduction in ablated depth when the removed volume is increased, showing that the crater becomes more and more "elongated". We also notice, however, the important exception of $\mathrm{Al}$ targets.

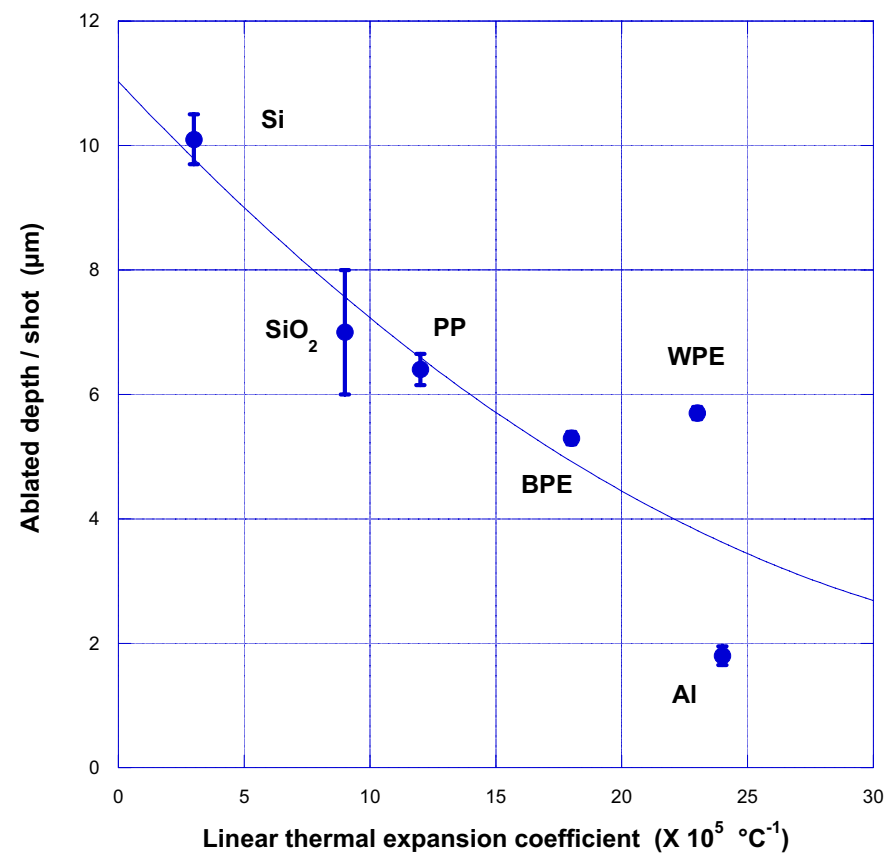

Fig. 6Ablated depth per laser shot vs. the linear thermal expansion coefficient

\section{Conclusions}

In this paper we have investigated the relation between material properties and laser crater morphology by means of a FIB/SEM system. We have shown how crater size and crater shape change with the sample material even with the same laser intensity, spot and pulse duration. The bulk modulus affects the crater sizes as described in ref [2]. Other thermal properties of the material seem instead to affect the shape of the carter (in particular from circular to elliptical) and change the morphology of craters highlighting thermal laser ablation processes. The observed relation between thermal properties and crater size and morphology could open the way to possible applications (e.g. in material recognition).
Of course, we do not claim that we have a complete and satisfactory theory of the phenomenon of crater formation. We just show that the carter shape formation is indeed a complex phenomenon and want to point out to some positive correlations. Predicting the topography generated by short (fs and ps) laser pulses is however a very challenging task. We think that our paper gives a contribution to the present field by showing that: under the same irradiation conditions crater morphology can be different for different materials, and several material and thermal parameters play a role in crater formation (and not only Young's modulus as it is expected on the basis of hydro scaling).

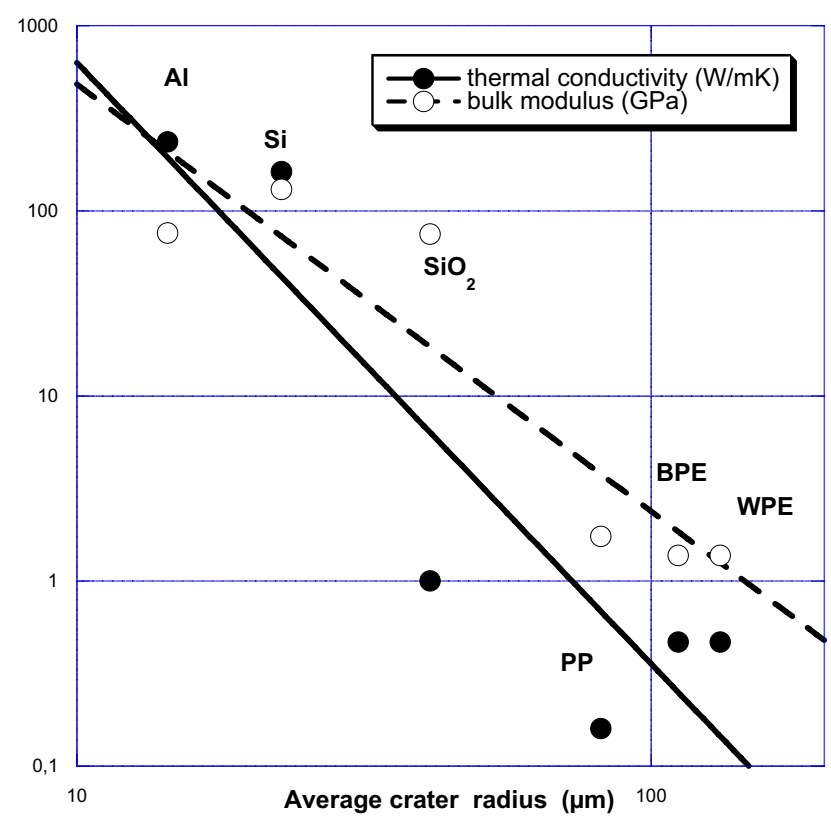

Fig. 7 Correlation between the average crater radius, bulk modulus and thermal conductivity of materials

\section{References}

[1] D.Batani "Short-pulse laser ablation of materials at high intensities: Influence of plasma effects" Laser and Particle Beams, Published online by Cambridge University Press 23 March 2010.

[2] E. G. Gamaly, V.Tikhoncuk, et al. "Laser matter interaction in the bulk of a transparent solid: Confined microexplosion and void formation" Physics Review E, 73 (2006) 21410.

[3] Y. P. Raizer et al, Laser induced Discharge Phenomena (1977).

[4] D. Bleiner, A. Bogaertes, Spectr. Act. Part B, 61 (2006) 421.

[5] M. Bussoli, D. Batani, M. Milani et al. "Study of laser induced ablation with focused ion beam/scanning electron microscope devices" Laser and Particle Beams, 25 (2007) 121.

[6] S. Petrovi, B. Gakovic, D. Perukoa, D. Batani, M. Cekadac, B. Radaka, and M. Trtica "Picosecond Laser Ablation of Nano Sized WTi Thin Film" Laser Physics, 19 (2009) 1844. 
[7] M. Milani et al. "An atlas of FIB/SEM in soft materials and life science" (Aracne Editrice, Rome, 2006).

[8] N. Sanner, O. Utéza, B. Bussiere, G. Coustillier, A. Leray, T. Itina, M. Sentis "Measurement of femtosecond laser-induced damage and ablation thresholds in dielectrics" Appl Phys A, 94 (2009) 889.

[9] Tables of Thermal properties of polymers (on line, e.g. http://wikipedia.com)

[10] H.R. Shanks et al, Thermal Conductivity of Silicon from 300 to 1400 K, Physical Review B, 130 (1963) 5.

[11] Eva Kuzielová et al. "Thermal stability of $\mathrm{Li}_{2} \mathrm{O}$ $\mathrm{SiO}_{2}-\mathrm{CaO}-\mathrm{P}_{2} \mathrm{O}_{5}-\mathrm{F}$ glass" Journal of Thermal Analysis and Calorimetry, 94 (2008) 849.

[12] J.M. Encinar and J.F. González "Pyrolysis of synthetic polymers and plastic wastes. Kinetic study" Fuel Processing Technology, 89 (2008) 678.

[13]A.Di Bernardo, D.Batani, C.Courtois, B.Cros, G.Matthieussent "High Intensity Ultra short laser induced ablation of metal targets in the presence of ambient gas" Laser and Particle Beams, 21 (2003) 59.

[14]D.Batani, H.Stabile, A.Ravasio, G.Lucchini, J.Ullschmied, E.Krousky, L.Juha, J.Skala, B.Kralikova, M.Pfeifer, C.Kadlec, T.Mocek, A.Präg, H.Nishimura, Y.Ochi "Ablation Pressure Scaling at Short Laser Wavelength“" Physical Review E, 68 (2003) 067403.

[15] Ya B. Zel'dovich and Yu P. Raizer "Physics Of Shock Waves And High-Temperature Hydrodynamic Phenomena" (Dover Publications, 2002) 